\title{
Severe Thrombocytopenia Associated With Black Seed Oil and Evening Primrose Oil
}

\author{
Xuejun Wang ${ }^{1}$, Alicia Jiang ${ }^{1}$, Vivek Batra ${ }^{2}$ \\ 1. Internal Medicine, Thomas Jefferson University Hospital, Philadelphia, USA 2. Medical Oncology, Thomas Jefferson \\ University Hospital, Philadelphia, USA
}

Corresponding author: Alicia Jiang, axj059@jefferson.edu

\begin{abstract}
We report the case of a 69-year-old female with stage IIIB endometrial adenocarcinoma who developed an acute thrombocytopenia with greater than $90 \%$ decrease from her baseline value in platelets one day after a laparoscopic hysterectomy. Subsequently, the patient was found to have bilateral subsegmental pulmonary emboli and a right atrial thrombus. The thrombocytopenia reached a nadir of $31,000 / \mathrm{mL}^{3}$ from a baseline of $410,000 / \mathrm{mL}^{3}$ and resolved without intervention. Prior to the surgery, the patient was taking black seed oil, which is commonly used for its anti-carcinogenic effects, and evening primrose oil daily for approximately one month. A literature review revealed that black seed oil contains thymoquinone, which is a compound related to quinine. Evening primrose oil is also known to reduce platelet aggregation and has antithrombotic properties. We believe the patient's thrombocytopenia was caused by a consumptive coagulopathy due to the formation of multiple thrombi and exacerbated by the use of herbal supplements, namely black seed and evening primrose oil.
\end{abstract}

Received 05/05/2020

Review began 05/12/2020 Review ended 05/21/2020 Published 06/01/2020

(c) Copyright 2020

Wang et al. This is an open access article distributed under the terms of the Creative Commons Attribution License CC-BY 4.0., which permits unrestricted use, distribution, and reproduction in any medium, provided the original author and source are credited.
Categories: Internal Medicine, Hematology

Keywords: primrose oil, nigella sativa, supplements, consumptive thrombocytopenia, drug-induced

thrombocytopenia, black seed oil, postoperative thrombocytopenia

\section{Introduction}

Thrombocytopenia is defined as a platelet count $<150,000 / \mathrm{mL}^{3}$ (normal range: $150,000-450,000 / \mathrm{mL}^{3}$ ), and is classified as mild (platelet count $\left.100,000-150,000 / \mathrm{mL}^{3}\right)$, moderate $\left(50,000-99,000 / \mathrm{mL}^{3}\right)$, or severe (< $\left.50,000 / \mathrm{mL}^{3}\right)$. The major complication of thrombocytopenia is bleeding. Spontaneous bleeding typically occurs when platelet counts are $<10,000 / \mathrm{mL}^{3}$. However, the correlation between bleeding and platelet count is highly variable. Etiologies of thrombocytopenia can be broadly categorized into decreased platelet production, increased platelet consumption, and sequestration of platelets [1].

The treatment of thrombocytopenia involves addressing the underlying cause. In deep venous thrombosis (DVT) and pulmonary embolism (PE), the mechanism of thrombocytopenia is related to increased platelet consumption due to the formation of thrombi. In drug-induced immune thrombocytopenia (DITP), medications or supplements lead to the production of antiplatelet antibodies and result in increased platelet consumption, and treatment typically involves discontinuing the offending drug. Once the drug is stopped, platelet counts typically recover within one to two weeks without further intervention.

Although there is no evidence for treating DITP with immunosuppressive therapy such as intravenous immunoglobulin (IVIG) and steroids, in clinical practice, IVIG and steroids are frequently given when there is concern for bleeding because immune thrombocytopenia (ITP) is difficult to differentiate from DITP. Furthermore, platelet transfusions are given to prevent bleeding if platelets are $<10,000 / \mathrm{mL}^{3}$ and $<50,000 / \mathrm{mL}^{3}$ for surgical procedures. In this case report, we present a patient with significant acute postoperative thrombocytopenia after using black seed oil and primrose oil daily for one month. This case report highlights thrombocytopenia as a potential adverse side effect of black seed and primrose oil supplements $[2,3]$. Written informed consent was obtained from the patient for publication of this case report.

\section{Case Presentation}

The patient is a 69-year-old African American female who was transferred to our facility for the management of anticoagulation in the setting of new bilateral PEs and thrombocytopenia. Prior to the transfer, the patient underwent a total abdominal hysterectomy with bilateral salpingo-oophorectomy for suspected endometrial cancer. She was subsequently diagnosed with stage IIIB serous endometrial adenocarcinoma based on surgical pathology. Her postoperative course was complicated by a profound thrombocytopenia and bilateral subsegmental PE without right ventricular strain. Her prior medical history includes a previous right lower extremity DVT with a right lower lobe PE. They occurred two months before her endometrial carcinoma diagnosis, likely in the context of a hypercoagulable state. She had an inferior 


\section{Cureus}

vena cava (IVC) filter placed and was eventually transitioned to apixaban for anticoagulation. Prior to the surgery, her home medications included apixaban $5 \mathrm{mg}$ twice a day, amlodipine $10 \mathrm{mg}$ daily, and acetaminophen $325 \mathrm{mg}$ as needed for pain. She also took herbal supplements daily, including black-seed oil and primrose oil, for approximately one month prior to the surgery.

The patient had a baseline platelet count of $410,000 / \mathrm{mL}^{3}$, which was measured two weeks prior to the surgery, and a baseline hemoglobin of $12 \mathrm{~g} / \mathrm{dl}$. The patient had no medication or clinical changes between her last lab draw and her surgical procedure. Given that her most recent labs were stable and the patient had no changes during the time frame, there was minimal concern for lab abnormalities and labs were not drawn just prior to surgery. After the surgical procedure, her platelet count decreased to $37,000 / \mathrm{mL}^{3}$ on postoperative day 1 . The estimated blood loss during the surgery was $600 \mathrm{~mL}$, for which she was transfused 2 units of packed red blood cells and 1 unit of pooled platelets. However, the patient continued to have worsening thrombocytopenia after the surgery, with a nadir of $31,000 / \mathrm{mL}^{3}$ on postoperative day 1 .

Anticoagulation was held until her platelets increased to $60,000 / \mathrm{mL}^{3}$ on postoperative day 5 . Initially, she was placed on a heparin drip and then transitioned to therapeutic enoxaparin for long-term anticoagulation. Her platelets returned to her previous baseline of $400,000 / \mathrm{mL}^{3}$ in approximately two weeks without any additional intervention.

Her thrombocytopenia was extensively worked up and multiple etiologies of secondary thrombocytopenia were considered, including heparin-induced thrombocytopenia, hemolysis, and viral-associated and thyroidrelated etiologies. The results of the work-up are outlined in Table 1. Her lab results were significant for postoperative hemoglobin of $10.5 \mathrm{~g} / \mathrm{dL}$ and white blood cell count of 11.4 per microliter.

\begin{tabular}{|c|c|}
\hline Study & Result \\
\hline \multicolumn{2}{|l|}{ Heparin-induced thrombocytopenia work-up } \\
\hline Serotonin-release assay & Non-reactive \\
\hline Platelet factor 4 & Non-reactive \\
\hline Peripheral smear & No schistocytes \\
\hline Haptoglobin & 194 mg/dl \\
\hline Lactate dehydrogenase (LDH) & 291 units/L \\
\hline Disseminated intravascular coagulation (DIC) panel & Negative \\
\hline Fibrinogen & 329 mg/dl \\
\hline D-dimer & $33,000 \mathrm{ng} / \mathrm{ml}$ \\
\hline \multicolumn{2}{|l|}{ Antiphospholipid syndrome (APLS) work-up } \\
\hline Anticardiolipin antibodies & Negative \\
\hline B2 glycoprotein antibodies & Negative \\
\hline Dilute Russell's viper venom time (dRVVT) & Negative \\
\hline HIV screen & Negative \\
\hline Hepatitis B screen & Negative \\
\hline Hepatitis C screen & Negative \\
\hline Thyroid-stimulating hormone (TSH) & $1.64 \mathrm{mlU} / \mathrm{L}$ \\
\hline
\end{tabular}

TABLE 1: Work-Up for Secondary Thrombocytopenia

\section{Discussion}

In this case, the patient had an acute decrease in platelets from a baseline of 410,000 platelets $/ \mathrm{mL}^{3}$ prior to surgery to a nadir of 31,000 platelets $/ \mathrm{mL}^{3}$ on postoperative day 1 . The patient had started taking black seed and primrose oil supplements about one month prior to the surgery. Her recorded baseline of 410,000 
platelets $/ \mathrm{mL}^{3}$ occurred two weeks after she started taking those supplements. Given the patient's work-up for secondary thrombocytopenia was negative, multiple etiologies could have contributed to this patient's thrombocytopenia in the postoperative setting. Postoperative thrombocytopenia and consumptive thrombocytopenia from the formation of multiple venous thromboembolisms likely caused the patient's acute thrombocytopenia. However, animal studies have also shown that ingestion of black seed oil or evening primrose oil decreased platelet counts in laboratory settings [2,3]. It is possible that the herbal supplements also partially contributed to the patient's thrombocytopenia following her surgery.

Surgery alone can cause thrombocytopenia via endothelial cell dysfunction and tissue factor and von Willebrand factor release leading to platelet activation and consumption. Early-onset postoperative thrombocytopenia is usually mild and resolves spontaneously within three to four days without bleeding [4]. One study that evaluated platelet counts in patients undergoing orthopedic surgery found that $28 \%$ of patients became thrombocytopenic after surgery, with only one patient having a platelet level $<100,000 / \mathrm{mL}^{3}$ postoperatively [5].

Acute thrombocytopenia can also occur from consumption related to an acute thrombus within 12 hours. An acute decrease in platelet count occurs following a DVT/PE because fresh thrombi exude thromboplastic substances and cause platelets to adhere to the thrombi's surface. The thrombocytopenia typically resolves spontaneously within three days [4]. In one prospective study of 189 patients with acute PE, the mean platelet decrease was $33,000 / \mathrm{mL}^{3}$, from $290,000-256,000 / \mathrm{mL}^{3}$ [6]. Similarly, the Urokinase Pulmonary Embolism Trial found the mean platelet count in acute PE patients was $293,000 / \mathrm{mL}^{3}[7]$. Another study enrolling patients with acute $\mathrm{PE}$ found admission platelet count to be $221,000 / \mathrm{mL}^{3}$ [8]. In contrast, one study found that patients who developed postop DVT, but not PE, had higher platelet counts than their preoperative levels [9].

Nigella sativa, or black-seed oil, is a popular herbal supplement that is frequently marketed as a "miracle supplement”. It has been studied for its beneficial effects, including anti-carcinogenic, anti-oxidant, antiinflammatory and analgesic, anti-diabetic, and antimicrobial effects [10]. Side effects of Nigella sativa are understudied, though the few studies available have shown low toxicity [11]. The main component of Nigella sativa is thymoquinone, a quinine constituent $[10,11]$. Quinine is a well-known cause of DITP, and there are many case reports of thrombocytopenia associated with quinine-containing beverages and over-the-counter medications, as well as a systematic review that revealed those who experienced DITP secondary to quinine had a median nadir platelet count of 3,000/ $\mathrm{mL}^{3}$ [12-14]. Animal studies on black-seed oil's effects on platelets have shown that oral administration of the fixed oil for 12 weeks decreased platelet counts by approximately $15 \%-35 \%$, compared to control values [2]. Another study proposed that thymoquinone induces apoptosis of platelets cells via a GPCR family receptor [15].

A wide variety of agents, including medications and many herbal substances, can cause the secondary form of ITP referred to as DITP. In addition to quinine, an analysis of case reports found 25 herbal substances and alternative medicines associated with thrombocytopenia. Many of the case reports reported platelet values as low as $0-16,000 / \mathrm{mL}^{3}$, although many of the patients did not experience thrombocytopenia alone [13]. The clinical course of DITP involves platelet counts decreasing in a week or longer after the drug is initiated depending on the frequency and quantity used [16]. Nadir platelet values often are $<20,000 / \mathrm{mL}^{3}$ and the clinical manifestations range from petechia to more severe presentations, such as microangiopathic hemolytic anemias. Specific treatment is rarely needed in DITP, as it typically resolves within a few days of drug discontinuation [16]. Thymoquinone is a compound that is related to quinine and it is possible that they share a similar mechanism that leads to thrombocytopenia, although less literature is available on thymoquinone $[10,11]$.

Evening primrose oil, an oil rich in $\gamma$-linolenic acid and omega-6-essential fatty acids, has been studied for its ability to affect platelet function. Studies in humans, rabbits, and rats have shown a decrease in platelet aggregation after ingestion of evening primrose oil [17-19]. Evening primrose oil's anti-platelet effect may stem from its ability to inhibit thromboxane B2, increase synthesis of other prostaglandins with weaker aggregation effects, and reduce hyperlipidemia [17-19]. Although there is less literature on evening primrose oil's effects on platelet count, one study in rabbits reported a significant decrease in platelet count after ingestion of evening primrose oil capsules for 60 days [3]. It has also been reported that evening primrose oil may increase the risk of bleeding in those concurrently taking anti-thrombotics [20].

\section{Conclusions}

In this case report, we highlight the role that black seed and primrose oil might have had in the patient's clinical presentation. The patient's thrombocytopenia was likely multifactorial with postoperative thrombocytopenia and consumptive thrombocytopenia as contributors. However, this acute drop in platelets was more significant compared to the typical values for postoperative and DVT/PE associated consumptive thrombocytopenia. The work-up for other causes of thrombocytopenia was largely negative, namely HIT, viral, thyroid, and autoimmune etiologies. Given black seed and evening primrose oil's effects on platelets, we propose that the use of herbal supplements also contributed to the development of severe 
thrombocytopenia in the postoperative setting. Therefore, it is important to consider the contribution of herbal supplements when evaluating and treating a patient with postoperative and consumptive thrombocytopenia, especially when the work-up for other secondary causes is negative.

\section{Additional Information \\ Disclosures}

Human subjects: Consent was obtained by all participants in this study. Conflicts of interest: In compliance with the ICMJE uniform disclosure form, all authors declare the following: Payment/services info: All authors have declared that no financial support was received from any organization for the submitted work. Financial relationships: All authors have declared that they have no financial relationships at present or within the previous three years with any organizations that might have an interest in the submitted work. Other relationships: All authors have declared that there are no other relationships or activities that could appear to have influenced the submitted work.

\section{References}

1. Izak M, Bussel JB: Management of thrombocytopenia. F1000Prime Rep. 2014, 6:45. 10.12703/P6-45

2. Zaoui A, Cherrah Y, Mahassini N, Alaoui K, Amarouch H, Hassar M: Acute and chronic toxicity of nigella sativa fixed oil. Phytomedicine. 2002, 9:69-74. 10.1078/0944-7113-00084

3. Riaz A, Khan RA, Ahmed SP: Assessment of anticoagulant effect of evening primrose oil . Pak J Pharm Sci. 2009, 22:355-359.

4. Ali N, Auerbach HE: New-onset acute thrombocytopenia in hospitalized patients: pathophysiology and diagnostic approach. J Community Hosp Intern Med Perspect. 2017, 7:157-167. 10.1080/20009666.2017.1335156

5. Zacharia G, Walczyszyn BA, Lee D, Stoffels G, Spaccavento C, Levine RL: Characteristics of the post-surgical decrease in platelet counts in orthopedic patients. Blood. 2016, 128:2554. 10.1182/blood.V128.22.2554.2554

6. Monreal M, Lafoz E, Casals A, Ruiz J, Arias A: Platelet count and venous thromboembolism: a useful test for suspected pulmonary embolism. Chest. 1991, 100:1493-1496. 10.1378/chest.100.6.1493

7. Sautter RD: The urokinase-pulmonary embolism trial. JAMA. 1974, 227:1168-1169. 10.1001/jama.1974.03230230044026

8. Konstantinides S, Geibel A, Heusel G, Heinrich F, Kasper W: Heparin plus alteplase compared with heparin alone in patients with submassive pulmonary embolism. N Engl J Med. 2002, 347:1143-1150. 10.1056/NEJMoa021274

9. Monreal M, Moreno V, Martorell A, et al.: Predicting pulmonary embolism in post-operative patients with deep venous thrombosis of lower limbs. Ann Vasc Surg. 1987, 1:421-425. 10.1016/S0890-5096(06)60726-7

10. Srinivasan K: Cumin (cuminum cyminum) and black cumin (nigella sativa) seeds: traditional uses, chemical constituents, and nutraceutical effects. Food Qual Saf. 2018, 2:1-16. 10.1093/fasafe/fyx031

11. Ali BH, Blunden G: Pharmacological and toxicological properties of nigella sativa. Phytother Res. 2003, 17:299-305. 10.1002/ptr.1309

12. George JN, Raskob GE, Shah SR, et al.: Drug-induced thrombocytopenia: a systematic review of published case reports. Ann Intern Med. 1998, 129:886-890. 10.7326/0003-4819-129-11 Part 1-199812010-00009

13. Royer DJ, George JN, Terrell DR: Thrombocytopenia as an adverse effect of complementary and alternative medicines, herbal remedies, nutritional supplements, foods, and beverages. Eur J Haematol. 2010, 84:421429. 10.1111/j.1600-0609.2010.01415.x

14. Liles NW, Page EE, Liles AL, Vesely SK, Raskob GE, George JN: Diversity and severity of adverse reactions to quinine: a systematic review. Am J Hematol. 2016, 91:461-466. 10.1002/ajh.24314

15. Towhid ST, Schmidt E-M, Schmid E, et al.: Thymoquinone-induced platelet apoptosis. J Cell Biochem. 2011, 112:3112-3121.10.1002/jcb.23237

16. Aster RH, Bougie DW: Drug-induced immune thrombocytopenia. N Engl J Med. 2007, 357:580-587. 10.1056/NEJMra066469

17. Abo-Gresha NM, Abel-Aziz EZ, Greish SM: Evening primrose oil ameliorates platelet aggregation and improves cardiac recovery in myocardial-infarct hypercholesterolemic rats. Int J Physiol Pathophysiol Pharmacol. 2014, 6:23-36.

18. Guivernau M, Meza N, Barja P, Roman O: Clinical and experimental study on the long-term effect of dietary gamma-linolenic acid on plasma lipids, platelet aggregation, thromboxane formation, and prostacyclin production. Prostaglandins Leukot Essent Fatty Acids. 1994, 51:311-316. 10.1016/0952-3278(94)90002-7

19. De La Cruz JP, Martin-Romero M, Carmona JA, Villalobos MA, Sanchez de la Cuesta F: Effect of evening primrose oil on platelet aggregation in rabbits fed an atherogenic diet. Thromb Res. 1997, 87:141-149. 10.1016/s0049-3848(97)00113-8

20. Agbabiaka TB, Wider B, Watson LK, Goodman C: Concurrent use of prescription drugs and herbal medicinal products in older adults: a systematic review. Drugs Aging. 2017, 34:891-905. 10.1007/s40266-017-0501-7 